\title{
Validation of self reported smoking
}

\section{Rebagliato}

\section{The use of cotinine as a biomarker for exposure to smoking}

S elf reported smoking status has been widely used to assess detrimental health effects of smoking and to orient counselling and other preventive interventions. Self reporting, however, can be unreliable if the subject is under pressure because of social or medical disapproval. Furthermore, the quantity of smoke products actually inhaled and absorbed varies by the manner of smoking. Because of these difficulties, increased emphasis has been placed on measuring exposure through the use of biological markers to provide more accurate estimates of smoking status and of the dose received.

In the past two decades, an increasing number of epidemiological studies have used biomarkers in assessing tobacco smoke exposure from active and passive smoking. Biomarkers can be used to classify people as exposed or unexposed, identify deceivers (people misreporting their smoking status), or estimate relative degree of exposure.

Cotinine, a major metabolite of nicotine, is currently regarded as the best biomarker for exposure of active smokers and non-smokers to environmental tobacco smoke (ETS). ${ }^{1}$ As a marker cotinine has the advantage of being almost specific to tobacco. The few exceptions include occupational exposure to tobacco leaves and nicotine products, use of smokeless tobacco products, chewing of nicotine gum, and use of nicotine patches or other aids for smoking cessation. Low levels of nicotine have been found in diet vegetables, but their impact in cotinine levels can be regarded as insignificant.

Cotinine can be measured in blood (that is, in serum), urine, saliva, and hair. The average half life of cotinine in different body fluids in adults is approximately 20 hours, making it a good indicator of the integrated exposure over the previous two to three days.'

Studies comparing non-smokers and smokers have consistently found that measurement of cotinine in the urine, saliva, or serum can distinguish active smokers from non-smokers. Active smokers almost always have serum or saliva levels higher than $15 \mathrm{ng} / \mathrm{ml}$ and sometimes greater than $500 \mathrm{ng} / \mathrm{ml}$. Nonsmokers exposed to typical levels of ETS have cotinine levels of less than $1 \mathrm{ng} / \mathrm{ml}$, with heavy exposure to ETS producing levels in the $1-15 \mathrm{ng} / \mathrm{ml}$ range. ${ }^{2}$
A number of studies have used biomarkers to validate self reported smoking status. Self reported non-smokers who seem to be smokers on the basis of biochemical measurements are generally considered "deceivers" of their true smoking status. However, caution should be used in making quantitative comparisons of smoking misclassification across studies because of differences in cut off points for distinguishing smokers from non-smokers, in population sources (clinical settings compared with community based studies), denominators used for misclassification rates (smokers compared with non-smokers), analytical techniques (gas chromatography compared with radioimmunoassay), and gender and ethnic differences in nicotine metabolism and smoking habits (smoking behaviours that may affect nicotine intake).

The serum and saliva cotinine cut off levels for distinguishing smokers from non-smokers reported in studies have varied from $3 \mathrm{ng} / \mathrm{ml}$ to $45.5 \mathrm{ng} / \mathrm{ml}$, with sensitivity ranged from $77 \%$ to $100 \%$, and specificity ranged from $79 \%$ to $100 \%{ }^{3}$

The cut off between the current smokers and non-smokers must be somewhat arbitrary because there is an overlap between non-smokers who are highly exposed to ETS with occasional smokers or those who inhale very little smoke. Authors who have tried to eliminate all possible smokers from their cohorts have used lower cut off points. ${ }^{4}$ However, those studies that tried to identify regular smokers used higher cut off points. In the 1992 Report on Lung Cancer and Passive Smoking by the US Environmental Protection Agency (EPA), ${ }^{4}$ the cut off point recommended to distinguish current smoker from non-smoker was 10\% of the self reported current smoker mean cotinine level. Misclassified smokers with cotinine levels greater than $30 \%$ of the self reported smoker mean cotinine level were defined as regular smokers. The rest of misclassified smokers, those with cotinine levels between $10 \%$ and $30 \%$ of the self reported smoker mean cotinine level, were defined as occasional smokers.

In a summary of 11 studies in which questionnaire responses regarding smoking status were compared with cotinine measurements, ${ }^{5}$ the estimated misclassification rates (proportion of self reported non-smokers with increased cotinine levels indicative of active smoking) ranged from $0.9 \%$ to $9.8 \%$, the highest value observed in a sample of non-smokers from a clinical setting.

The discrepancy in misclassification rates between subjects of community based studies and those recruited in smoking cessation trials or clinical settings is also patent in specific subgroups of the population. For example, misclassification rates reported among pregnant women may be as different as 3\% in a population based survey ${ }^{6}$ and $26.2 \%$ in a smoking cessation trial. ${ }^{7}$

Gender, ethnic and social differences in misclassification rates have been observed in different studies. In the US Third National Health and Nutrition Examination Survey (1988-1994), 1.4\% of self reported non-smokers had a serum cotinine level greater than 15.0 $\mathrm{ng} / \mathrm{ml}$, the selected cut-off point for identifying non-smokers. Misclassification rates were higher among the black population and lower educated people. ${ }^{8}$

Wells et al ${ }^{9}$ calculated from data of 10 studies the combined misclassification rate (self reported non-smokers with cotinine levels above cut off point divided by the total number of self reported current smokers) stratified by sex, ethnic minority/majority status, and whether the misclassified smoker was considered occasional or regular smoker according to the EPA's criteria. ${ }^{4}$ The misclassification rate of female regular smokers misclassified as never smokers varied from $0.8 \%$ in the ethnic majority group to $2.8 \%$ in the minority group. The misclassification rate of female occasional smokers misclassified as never smokers varied from $6.0 \%$ in the ethnic majority group to $15.3 \%$ in the minority group. The misclassification rate pattern for men was similar but with slightly higher values. The authors concluded that although smoker misclassification bias is small, investigators are advised to pay attention to gender and minority/ majority status of cohorts when correcting for smoker misclassification bias.

Deception regarding smoking status may explain most of the discrepancy in misclassification rates between clinical settings and population based studies. This explanation, however, cannot be extrapolated to gender and ethnic differences. In this sense, Caraballo et al ${ }^{10}$ provided the first evidence from a national study that serum cotinine levels are higher among black smokers than among white or Mexican American smokers. These differences did not seem to be attributable to differences in ETS exposure or in number of cigarettes smoked. Higher levels of cotinine per cigarette smoked by the black population compared with the white popluation can be explained by both slower clearance of 
cotinine and higher intake of nicotine per cigarette in black people. ${ }^{11}$ Greater nicotine and therefore greater tobacco smoke intake per cigarette might, in part, explain why black people find it harder to stop smoking and some of the ethnic differences in smoking related disease risks.

Pregnancy status may also exert an effect on the uptake and metabolism of nicotine. Pregnant smoking women have lower cotinine levels than non-pregnant smokers. ' In the light of this observation, we studied in a sample of Spanish smoking pregnant women the differences in saliva cotinine values during and after pregnancy. ${ }^{12}$ After allowing for differences in smoking practices, cotinine per cigarette ratio during pregnancy was significantly lower that the ratio in the postnatal testing.

In this issue, the paper of Vartiainen et $a l^{13}$ adds further evidence to the high validity of self reported smoking observed in population based studies. Serum cotinine concentrations determined by gas chromatography were used to validate self reported smoking in a community-based cardiovascular prevention programme (North Karelia project) and in other areas of Finland involved in the WHO MONICA survey. A cut off point of $10 \mathrm{ng} / \mathrm{ml}$ was used. The overall misclassification rate among those who reported not having smoked in the past month was $6.3 \%$ in men and $5.2 \%$ in women. Among never smokers $2.5 \%$ of men and $2.7 \%$ of women had cotinine levels of at least $10 \mathrm{ng} / \mathrm{ml}$. No statistically significant differences in misclassification rates were observed between age, sex, marital status, educational groups, or areas of residence.

The authors make the point that smoking patterns are changing in populations, from a more clearly defined habit-people were either smokers or non-smokers-to an increasing number of occasional smokers. In this survey, $20 \%$ of male smokers and $30 \%$ of female smokers reported that they smoked occasionally. About half of the self reported occasional smokers had cotinine values lower than $10 \mathrm{ng} / \mathrm{ml}$. Classic calculations of sensitivity and specificity may therefore not be as appropriate as they were when occasional smoking was infrequent. On the other hand, high cotinine values among non-smokers might be partly explained by the use of nicotine replacement therapy or other nicotine delivery products such as chewing gum or smokeless tobacco. The authors summarise that, as occurs in other surveys, the number of misclassified people is small, and "this raises the question of whether costly biochemical validation procedures are needed in population based surveys."

As the authors conclude, validity of self reported smoking is consistently high in population based studies and therefore the extended use of cotinine measurements for validation purposes may not be justified. Nevertheless, further research may focus on assessing the optimal cut off point for validating smoking status among specific groups, such as pregnant women, ethnic groups, experimenters or occasional smokers These studies will also improve our understanding of the effects of gender, ethnicity, social conditions, and pregnancy status on the metabolism of nicotine and on smoking behaviours that may affect nicotine intake.

Finally, biomarkers can be used to assess not only the validity of people's self reporting of smoking but also the validity of regulators' methods to control tobacco products. Jarvis et al ${ }^{14}$ suggested that smoking machines used to characterise the tar and nicotine yields of cigarettes actually underestimate the true intake of these chemicals and should be abandoned. At any level of nicotine yield, the researchers found no agreement in the nicotine intake per cigarette smoked estimated from salivary cotinine level and that derived from machine smoked yields. In fact, for the lowest yield cigarettes, the smoking machine estimated nicotine levels that were eight times lower than estimates from salivary cotinine levels. These results once more calls into question the magnitude of the potential reduction in health risk obtained by smoking low tar and nicotine brands, and emphasise the need of developing better measurements of the constituents and impact of tobacco products with the aim of substantially reducing their toxicity. J Epidemiol Community Health
2002;56:163-164

\section{Authors' affiliations}

M Rebagliato, Departamento de Salud Pública, Universidad Miguel Hernández, San Juan (Alicante), Ap Correo 374, 03080, Alicante, Spain

Correspondence to: Dr M Rebagliato; rebagli@umh.es

\section{REFERENCES}

1 Benowitz NL. Cotinine as a biomarker of environmental tobacco smoke exposure. Epidemiol Rev 1996;18:188-204.

2 Centers for Disease Control and Prevention. National report on human exposure to environmental chemicals. Atlanta: Centers for Disease Control and Prevention,

National Center for Environmental Health: 2001:33. NCEH Pub no 01-0379:33.

3 Patrick DL, Cheadle A, Thompson DC, et al. The validity of self-reported smoking: a review and meta-analysis. Am J Public Health 1994;84:1086-93

4 Wells AJ. Appendix B: Method for correcting relative risk for smoker misclassification. In: US. Environmental Protection Agency. Respiratory health effects of passive smoking: lung cancer and other disorders. Washington, DC: EPA Office of Research and Development, 1992. EPA/600/6-90/006F.

5 Perez-Stable EJ, Marin G, Marin BV, et al. Misclassification of smoking status by self-reported cigarette consumption. Am Rev Respir Dis 1992;145:53-7.

6 Owen L, McNeill A. Saliva cotinine as indicator of cigarette smoking in pregnant women. Addiction 2001;96:1001-6.

7 Boyd NR, Windsor RA, Perkins LL, et al. Quality of measurement of smoking status by self-report and saliva cotinine among pregnant women. Matern Child Health $J$ 1998;2:77-83.

8 Caraballo RS, Giovino GA, Pechacek TF, et al. Factors associated with discrepancies between self-reports on cigarette smoking and measured serum cotinine levels among persons aged 17 years or older. Third National Health and Nutrition Examination Survey, 1988-1994. Am J Epidemiol $2001 ; 153: 807-14$

9 Wells AJ, English PB, Posner SF, et al. Misclassification rates for current smokers misclassified as nonsmokers. Am J Public Health 1998; 88:1503-9.

10 Caraballo RS, Giovino GA, Pechacek TF, et al. Racial and ethnic differences in serum cotinine levels of cigarette smokers. Third National Health and Nutrition Examination Survey, 1988-1991. JAMA 1998:280:135-9.

11 Pérez-Stable EJ, Herrera B, Jacob III P, et al. Nicotine metabolism and intake in black and white smokers. JAMA 1998;280:152-6.

12 Rebagliato M, Bolúmar F, Florey $C V$, et al. Variations in cotinine levels in smokers during and after pregnancy. Am J Obstet Gynecol 1998;178:568-71.

13 Vartiainen E, Seppälä T, Lillsunde P, et al. Validation of self reported smoking by serum cotinine measurement in a community-based study. J Epidemiol Community Health 2002;56:167-70.

14 Jarvis MJ, Boreham R, Primatesta P, et al. Nicotine yield from machine-smoked cigarettes and nicotine intakes in smokers: evidence from a representative population survey. Natl Cancer Inst 2001;93:134-8. 